\title{
Serological detection of brucellosis among febrile, malaria-negative children and domesticated dogs in an urban African setting
}

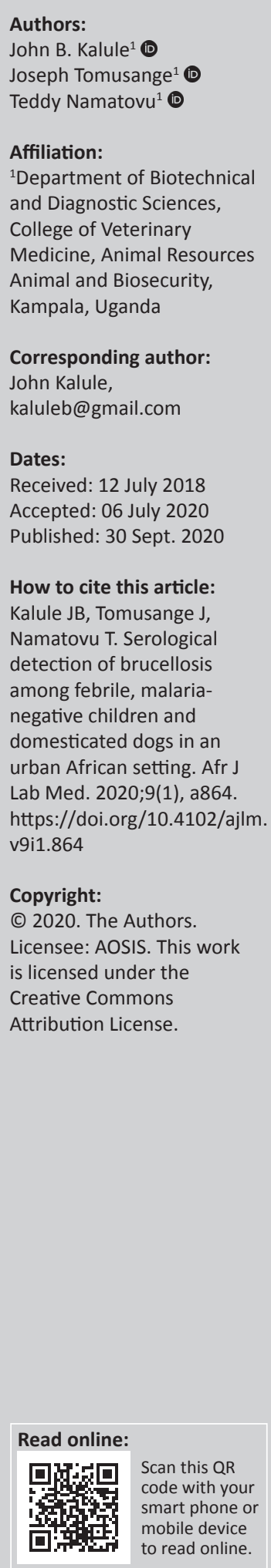

Background: Childhood brucellosis and malaria are co-endemic febrile illnesses in some subSaharan African countries. Malaria and brucellosis co-infection or brucellosis sole infections are often missed due to an over emphasis on malaria and the lack of appropriate diagnostic infrastructure. Brucellosis in dogs is usually overlooked and yet there is extensive contact between humans and their pets.

Objective: This study investigated brucellosis in children and dogs using a confirmatory serological testing series that screens for three Brucella sp.

Methods: Residual blood samples from malaria smear-negative febrile children were collected and tested for Brucella sp and malaria parasite. During the same period, residual blood samples presented to a veterinary microbiology laboratory in the same area were tested for brucellosis using the same approach.

Results: A total of 105 human and 80 canine blood samples were tested for brucellosis antibodies. The seroprevalence of brucellosis was $22.86 \%(25 / 105)$ in children and $1.3 \%(1 / 80)$ in dogs using the Card, buffered acidified plate antigen, and standard plate agglutination tests but was $0 \%$ using the rivanol precipitation plate agglutination test.

Conclusion: Given that brucellosis can be caused by both smooth and rough colony strains, there is a need to modify the current serological surveillance strategy (targeted at only Brucella abortus and other smooth colony Brucella strains) to figure out the relative contribution of rough colony Brucella strains (B. ovis and B. canis). Since Uganda is endemic for brucellosis there is a need to modify the brucellosis surveillance strategy.

Keywords: brucellosis; serology; malaria; febrile illness; diagnostics.

\section{Introduction}

Globally, human brucellosis remains the most common zoonotic disease with more than 500000 new cases annually, most of which are in sub-Saharan Africa. ${ }^{1}$ Generally, brucellosis has no age predilection; its transmission is linked to use of or contact with known sources of infection, such as consumption of poorly made dairy products, ${ }^{2,3}$ or contact with carcasses or aborted material of infected livestock. Childhood brucellosis has previously been reported among children presenting with pyrexia of unknown origin. ${ }^{4}$ Childhood brucellosis accounts for up to one-third of all cases of human brucellosis in endemic regions. ${ }^{5}$

Uganda is one of the sub-Saharan Africa countries that are endemic for brucellosis and have, according to World Organisation for Animal Health (OIE) data, reported cases of human brucellosis. ${ }^{6}$ As per a 2006 study which estimated brucellosis globally, Uganda had an estimated incidence of 1.8 new cases per 100000 population. $^{7}$

Both the vaccine strain (Brucella abortus S19) and the wild strains (B. abortus, B. melitensis, B. canis and $B$. suis) have been shown to be excreted in substantial amounts in livestock milk, ${ }^{8,9,10}$ a wholesome nutritious option for weaned children, ${ }^{11}$ thus milk is a vehicle of brucellosis transmission to humans. ${ }^{12}$ Milk and/or milk products should be subjected to various physical sterilisation to kill potential pathogens such as Brucella spp. ${ }^{13}$ However, due to various socioeconomic factors, consumption of milk and milk products remains a risk factor for brucellosis in many regions in sub-Saharan Africa. ${ }^{10,14,15,16,17}$ Nevertheless, other risk factors, such as environmental contamination with aborted foetal material, or contact with infected domesticated dogs or their body fluids, could potentially be associated with brucellosis, ${ }^{18}$ even among 
children. Dogs have been proven to be reservoirs of highly pathogenic canine $B$. suis strains that can cause severe disease in humans ${ }^{19}$ and/or re-emergence of brucellosis on livestock farms. ${ }^{20}$

The clinical presentation of brucellosis is largely similar to that of malaria (high fever, joint pain, malaise, headache and chills $)^{21}$; thus, there is a need to deploy sensitive, specific, rapid, and cost-effective laboratory diagnostic tools to differentiate them and avoid misdiagnosis. ${ }^{3}$ The situation is aggravated by the presence of the weak laboratory infrastructure in sub-Saharan Africa. ${ }^{22}$ Moreover, the morbidity and mortality rates associated with malaria are high, yet coinfections with brucellosis in this setting are not uncommon. ${ }^{3}$ Children presenting with high fevers, headaches, and joint pains are more likely to be treated empirically for malaria. ${ }^{23}$

The diagnosis of brucellosis requires the use of several clinical investigative techniques across haematology, biochemistry, radiology, bacteriology, serology and molecular biology. ${ }^{24}$ Isolation of Brucella spp. from the blood, bone marrow or other tissue fluids is the gold standard. ${ }^{5}$ Unlike most highincome countries where brucellosis affects mostly animals, in sub-Saharan Africa it is arguably endemic in both humans and animals..$^{25}$ In North America, where brucellosis affects both domestic (such as cattle and goats) and wild animals (such as elk and bison), highly efficient serological tests have been developed as part of surveillance diagnostic test schemes for both. ${ }^{26}$ Seven such serological tests, the Card (Rose Bengal) test, the complement fixation test, the rivanol precipitation plate agglutination (RPPA) test, the standard plate agglutination (SP) test (SPT), the buffered acidified plate antigen (BAPA) test, the rapid automated presumptive test, and the fluorescence polarisation assay have been approved by the United States Department of Agriculture for the detection of B. abortus antibodies. Brucella abortus is the most prevalent species in North America. The rapid slide agglutination test is often used to test for $B$. canis in dogs which can also be infected with B. suis. ${ }^{27}$ The B. abortus serological tests that are commonly used cannot detect antibodies to the rough colony variants B. canis and B. ovis. The North American serological tests have rarely been used in the endemic African regions; in fact, only the Card test has been used frequently for serodiagnosis of brucellosis at the point of care for both humans and animals. ${ }^{28}$ The Card test is highly efficient for diagnosis of human brucellosis, often outperforming tests that take longer to perform such as Coombs, competitive enzyme-linked immunosorption assay, BrucellaCapt (immunocapture agglutination test; Vircell, Granada, Spain), ${ }^{29}$ immunochromatography, and immunoprecipitation with Brucella proteins. ${ }^{30}$ With the exception of the RPPA and the fluorescence polarisation tests, the SPT, Card, rapid automated presumptive and BAPA tests all have the limitation that immunoglobulin $\mathrm{M}$ antibodies against Brucella spp. cross-react with those of other Gramnegative bacteria such as Salmonella spp. On the other hand, the immunoglobulin $\mathrm{G}$ antibodies that are detected using the RPPA test do not cross-react and thus give fewer false positives. Unlike seropositivity by Rose Bengal test or BAPA, which in addition to indicating infection may also mean recovery from brucellosis, RPPA test seropositivity can be used to differentiate between active infection or past infection. ${ }^{12}$

Most studies of Brucella spp. seroprevalence in Uganda have used the Rose Bengal test (Card test) without using a confirmatory serological test as categorised by the OIE. . $^{31,32,33}$ The RPPA test is commonly used as a confirmatory test, because the non-specific reactivity is reduced by precipitation of high molecular weight serum glycoproteins. ${ }^{12}$ The increased specificity means a reduction in the number of false positives. This study aimed to establish the seroprevalence of brucellosis due to B. abortus and B. suis in humans presenting with acute febrile illness and domesticated dogs in the same urban African setting. We hypothesised that the use of a confirmatory serological test (RPPA) in addition to routinely used screening serological tests (BAPA, Card and SPT) would enable us to rule out positive reactions due to vaccination of tested animals, previous infection history, or cross reactions ${ }^{34}$ and thus figure out the true seroprevalence of brucellosis due to B. abortus and B. suis in this setting.

\section{Methods \\ Ethical considerations}

Ethical clearance was obtained from the Makerere University, College of Veterinary Medicine, Animal Resources and Biosecurity (CoVAB), School of Biomedical and Biotechnical Laboratory Sciences, Research and Ethics Committee (SBLS/ REC/13/019). The ethical clearance was for use of residual blood samples from the paediatric ward at the clinical microbiology laboratory at Mulago National Referral Hospital. The same clearance also permitted the use of residual blood samples from dogs at a small animal veterinary clinic at Makerere University College of Veterinary Medicine Animal resources and Biosecurity. This study did not directly recruit or collect samples from either the febrile children or the febrile dogs.

Data on the ages and sex of the patients from whom the samples were collected were recorded. The samples were re-assigned a study number and all patient-identifying data were not accessed. The same was done for the residual blood samples from febrile dogs.

\section{Study design and residual sample selection}

We opted to test residual blood samples from febrile children that were malaria smear-negative and residual blood samples from febrile dogs. The dogs, though owned by individual homes, closely interacted with the community in the study region; for instance, the pig abattoir in the community is potentially a common source of infection to both humans (via meat purchases) and dogs (either the abattoir waste was purchased by the dog owner as dog feed or the dogs accessed poorly disposed abattoir waste). 
All samples used were collected between January and June 2014 (Figure 1). The malaria-negative residual paediatric blood samples were collected from the clinical microbiology laboratory of a regional referral hospital, while residual canine blood samples were from a local veterinary clinic which serves the central region. The residual blood samples were tested for brucellosis using three screening tests and one confirmatory test.

\section{Re-testing human blood samples for malaria}

The included malaria-negative paediatric residual blood samples were re-tested for malaria to confirm that they were malaria-negative. The Field's stains A and B (Himedia Laboratories Limited, Mumbai, India) were used to stain malaria parasites and were viewed using light microscopy. Briefly, a drop of blood was placed on a clean $25 \mathrm{~mm}$ x $75 \mathrm{~mm}$ glass slide (Merck, Darmstadt, Germany) and a thick smear was made at the centre of the glass slide using the edge of another glass slide to spread to an area of $1 \mathrm{~cm}^{2}$. The smear was allowed to dry at room temperature for $1 \mathrm{~min}$. It was then sequentially dipped into Field's stain A (Himedia Laboratories Limited, Mumbai, India) for 3 seconds and washed in de-ionised water for $3 \mathrm{~s}$ with gentle agitation, then it was dipped in Field's stain $\mathrm{B}$ for $3 \mathrm{~s}$ and washed in tap water for $5 \mathrm{~s}$. The stained smear was then air dried and examined under a light microscope at X100 magnification under oil immersion for malaria parasites, as previously described. ${ }^{35}$

\section{Testing of human and canine sera samples for Brucella abortus/suis antibodies}

Human blood samples that were negative for malaria were processed by centrifugation and the serum obtained was tested for brucellosis using: the Becton Dickinson Brucellosis Card Test Kit 306® (National Veterinary Services Laboratory [NVSL], Ames, Iowa, United States) - a screening test for antibodies against B. abortus/B. suis in serum and plasma; SPT (NVSL, Ames, Iowa, United States); BAPA (SL, Ames,

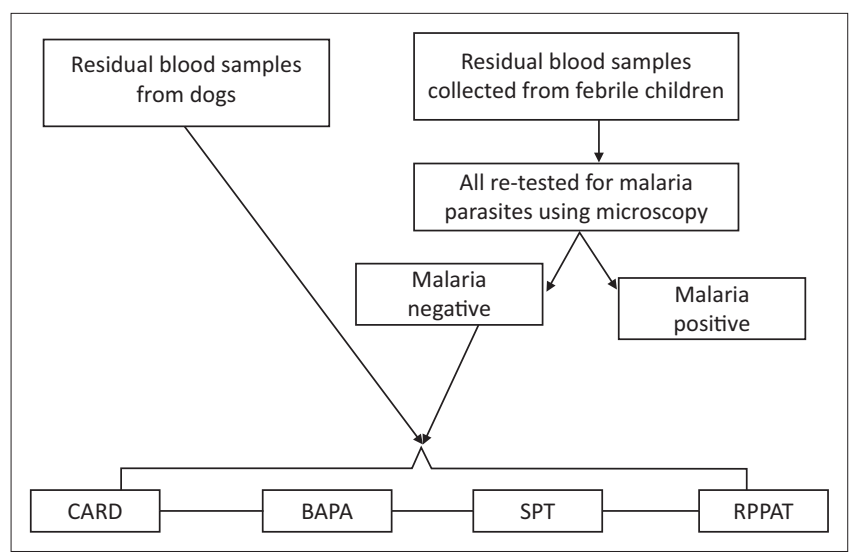

Note: Study design and methods for serological testing of humans and dogs tested between January and June 2014 in Kampala, Uganda

BAPA, buffered acidified plate antigen test; CARD, brucellosis Card test; SPT, standard plate agglutination test; RPPAT, rivanol precipitation plate agglutination test.

FIGURE 1: Sample testing algorithm for blood samples from children and dogs in Kampala, Uganda (January and June 2014).
Iowa, United States) and RPPA (NVSL, Ames, Iowa, United States) - a precipitation and agglutination test.

The BAPA, RPPA, Card and SP kits screened for B. abortus/ B. suis antibodies in serum and plasma; tests were carried out according to the manufacturers' instructions on all human and canine sera included in the study. Except otherwise stated, the control sera used were the B. abortus complement fixation medium positive control serum (NVSL reagent 12-M) and the $B$. abortus complement fixation negative control serum (NVSL reagent 12-N).

\section{Buffered acidified plate antigen test}

Aseptically, $80 \mu \mathrm{L}$ of each sample or control serum was pipetted onto separate squares (1.25 inch -1.5 inch) etched on a 12.5-inch $\times 12.5$-inch white ceramic bioassay glass plate. Then $30 \mu \mathrm{L}$ of buffered Brucella plate antigen (NVSL, Ames, Iowa, United States) was added to each sample or control serum square. The serum-antigen suspension was then mixed using a glass stirrer to a diameter of approximately $27 \mathrm{~mm}$ and incubated for 4 minutes $( \pm 30 \mathrm{~s})$ at room temperature $\left(20^{\circ} \mathrm{C}-26^{\circ} \mathrm{C}\right)$ in an enclosed space. After this incubation, it was stirred again and incubated a second time at room temperature for $4 \mathrm{~min}$. The slides were then read in a Minnesota box - an illuminator with an indirect source of light, a black background, and a cover to prevent evaporation of test reagents.

\section{Rivanol precipitation plate agglutination test}

Aseptically, $200 \mu \mathrm{L}$ of each sample or control (positive or negative) was transferred into respective (well-labelled) tubes. Thereafter, $200 \mu \mathrm{L}$ of Brucella rinavol solution was transferred into each tube and mixed by shaking for $1 \mathrm{~min}$. The mixture was incubated at room temperature for $5 \mathrm{~min}$ for precipitation. The tubes were centrifuged at 3000 revolutions per minute to pellet the precipitates. Aseptically, $80 \mu \mathrm{L}, 40 \mu \mathrm{L}, 20 \mu \mathrm{L}$ and $10 \mu \mathrm{L}$ of each sample or control supernatant were dispensed onto separate squares (1.25 inch - 1.5 inch) etched on a 12.5 inch $\times 12.5$ inch white ceramic bioassay glass plate. Then, $30 \mu \mathrm{L}$ of B. abortus rivanol antigen was added. The serum-antigen mixture was stirred in circles (at least 8 circles) beginning from the 1:200 serum dilutions, then proceeding to the 1:25 dilutions of sample or control sera. The bioassay plate was then incubated at room temperature $\left(25 \pm 2{ }^{\circ} \mathrm{C}\right)$ in an enclosed space for $6 \mathrm{~min}( \pm 30 \mathrm{~s})$ with rotation. The incubation with rotation was repeated for a further $6 \mathrm{~min}( \pm 30 \mathrm{~s})$. The reading was taken at the end of the second incubation under an illuminator according to the manufacturer's instructions.

\section{Card test}

Aseptically, $30 \mu \mathrm{L}$ of sample or control (positive or negative) were dispensed onto the reaction area on the card. Subsequently, two drops of the B. abortus Card test antigen (NVSL, Ames, Iowa, United States) were then dispensed onto the card adjacent to the sample. The antigen and serum were mixed using a clean wooden stirrer for approximately $15 \mathrm{~s}$, followed by rocking movements for $4 \mathrm{~min}$. 


\section{Standard plate agglutination tests}

Aseptically, $80 \mu \mathrm{L}, 40 \mu \mathrm{L}, 20 \mu \mathrm{L}$ and $10 \mu \mathrm{L}$ of sample or control serum were dispensed onto separate squares on a white ceramic bioassay glass plate. Following gentle mixing, $30 \mu \mathrm{L}$ of antigen was dispensed to each square containing sample or control serum. The serum and antigen were mixed with a stirrer in circles. Mixing was done beginning with the 1:200 dilution. Twice, consecutively, the glass plate was rocked through four rotations, followed by incubation at $25 \pm 2{ }^{\circ} \mathrm{C}$ in an enclosed space for $4 \mathrm{~min}( \pm 30 \mathrm{~s})$. The white ceramic bioassay glass plate was rotated again and incubated for another $4 \mathrm{~min}( \pm 30 \mathrm{~s})$. The reaction was then read using a Minnesota box (NVSL, Ames, Iowa, United States).

\section{Statistical analysis}

Data on the serological reactions were entered in Microsoft Office Excel 2010 (Microsoft Corp., Redmond, Washington, United States) and then exported to EPIINFO ${ }^{\mathrm{TM}}$ 7.1.5.2 (Centers for Disease Control and Prevention, Atlanta, Georgia, United States) for analysis to determine proportions. The OpenEpi diagnostic test evaluation calculator ${ }^{36}$ was used to estimate parameters for the performance of the different screening tests compared to the RPPA test.

\section{Results}

A total of 349 human residual blood samples were received at the clinical microbiology laboratory from the paediatric ward during the study period; 80 canine residual blood samples were received at the small animal veterinary clinic during the same time period. Of the 349 human samples, 105 were smear-negative for malaria; 244 were smear-positive and excluded from further analysis. Of the 105 malaria smear-negative samples, $44.8 \%(47 / 105)$ were from male patients and $55.2 \%(58 / 105)$ were from female patients. Seroprevalence of brucellosis among the malaria smear-negative residual blood samples was $23.8 \%(25 / 105)$ by Card, $23.8 \%(25 / 105)$ by BAPA and $23.8 \%(25 / 105)$ by SPT, but was $0 \%(0 / 105)$ using the RPPA test (Table 1). All the samples that tested positive using the Card test were also positive using the BAPA and the SP tests; therefore, there was a 100\% agreement between Card, BAPA and SP tests. Of the 25 samples that tested positive using the three screening tests, none $(0 \%, 0 / 25)$ were confirmed as positive using the RPPA test. Of the 25 human samples that were positive, $40 \%(10 / 25)$ were from

TABLE 1: Sero-reactions to brucellosis tests for human and canine blood samples tested between January and June 2014 in Kampala, Uganda.

\begin{tabular}{|c|c|c|c|c|c|c|c|c|}
\hline \multirow[t]{2}{*}{ Sero-reaction } & \multicolumn{2}{|c|}{ BAPA } & \multicolumn{2}{|c|}{ Card } & \multicolumn{2}{|c|}{ SPT } & \multicolumn{2}{|c|}{ RPPA } \\
\hline & $n$ & $\%$ & $n$ & $\%$ & $n$ & $\%$ & $n$ & $\%$ \\
\hline \multicolumn{9}{|c|}{ Humans $(n=105)$} \\
\hline+ & 25 & 23.8 & 25 & 23.8 & 25 & 23.8 & 0 & 0.0 \\
\hline - & 80 & 76.2 & 80 & 76.2 & 80 & 76.2 & 105 & 100.0 \\
\hline \multicolumn{9}{|l|}{ Dogs $(n=80)$} \\
\hline+ & 1 & 1.25 & 1 & 1.25 & 1 & 1.25 & 0 & 0.0 \\
\hline- & 79 & - & 79 & - & 79 & - & 80 & 100.0 \\
\hline
\end{tabular}

$+/-$, positive/negative; BAPA, buffered acidified plate antigen test; Card, brucellosis Card test; SPT, standard plate agglutination test; RPPA, rivanol precipitation plate agglutination test. male patients, and $60 \%(15 / 25)$ were from female patients. There was no significant difference between sero-reactions for male and female children $(p=0.131)$. The mean age of the seropositive children was $12( \pm 0.8)$ years.

Eighty blood samples from dogs (one sample from each dog), all from households in the central region, were tested for B. suis antibodies, and only one was positive using the three screening serological screening tests - Card, BAPA and SPT. Thus, the seroprevalence of canine B. suis was $1.28 \%(1 / 80)$ using the screening tests. This sample was, however, negative using the RPPA test (seroprevalence using the RPPA test $=0 \%$ ). There was no significant difference between the seropositivity for humans and dogs $(p=0.052)$.

Of the canine $(1 / 80)$ and human $(25 / 105)$ samples that tested positive by the SP test, all were positive at the two lowest dilutions (1:25 and 1:50). However, 60\% $(15 / 25)$ of the human samples tested positive at the third dilution (1:100) and $28 \%(7 / 25)$ tested positive at the fourth dilution (1:200).

For the human samples, the specificity of the screening tests (Card, BAPA and SPT) was 76.2\% (67.2-83.3), sensitivity $0 \%$, positive predictive value $0 \%$, and the negative predictive value $100 \%$. The Cohen's Kappa statistic was 0.

For the canine samples, the specificity of the screening tests (Card, BAPA and SPT) was 76.2\% (67.2-83.3), sensitivity $0 \%$, positive predictive value $0 \%$, and the negative predictive value $100 \%$. The Cohen's Kappa statistic was 0.

\section{Discussion}

This study used three screening tests (Card, BAPA and SP) and an OIE confirmatory test (RPPA) to evaluate brucellosis as a possible aetiology of pyrexia of unknown origin among malaria smear-negative febrile children in a malaria-endemic African region. Using routine serological screening tests, the seroprevalence of brucellosis in dogs was $1.3 \%$, and $23.8 \%$ in humans. There was 100\% agreement between Card, BAPA and SP tests, but all were negative using the confirmatory RPPA test. The seroprevalence of brucellosis in both species using the OIE-recommended confirmatory serological test RPPA test - was $0 \%$.

Many serological studies conducted on brucellosis in endemic regions have reported a high prevalence of brucellosis..$^{37,38,39,40}$ Unlike earlier studies, this study deployed an OIE confirmatory test in parallel with routinely used screening tests. The brucellosis prevalence reported without the use of a confirmatory test might be misleading, particularly when Brucella culture, the gold standard for Brucella spp. detection, is not carried out. Brucella culture is difficult to execute and suitable laboratory facilities are often lacking in resource-limited brucellosis-endemic regions. 
The discrepancy between the screening and confirmatory tests found in the current study is similar to that reported in a study conducted in Kenya. ${ }^{41}$

In this study, all the blood samples tested using the Card and other screening tests were negative when tested using the RPPA test. The difference in results can be explained by the fact that the most commonly used screening test in Uganda, the Card (Rose Bengal) test, deploys a B. abortus/suis antigen that would miss the detection of rough Brucella spp. such as B. canis and $B$. ovis. ${ }^{42}$ Also, cross-reaction is not uncommon when using Card (Rose Bengal), leading to false positives. The smooth lipopolysaccharide O-chain of smooth B. abortus, B. melitensis and B. suis and other Gram-negative bacteria expressing the lipopolysaccharide O-chain (such as Escherichia coli O157:H7 and Yersinia enterocolitica O:9) have been shown to cross-react with the Card (Rose Bengal) test (B. abortus/suis antigen). ${ }^{43}$ Also, the Card test does not differentiate between vaccinated and infected animals, or of the recovered individuals (as would be expected in a brucellosis-endemic setting) from those with active infection. $^{34}$ On the other hand, the RPPA test is a quantitative and specific test which is able to rule out false-positive serological reactions. ${ }^{34}$ These factors could account for the difference in results between the Card and the RPPA test.

Additionally, even though there was agreement between the three serological screening tests (Card, BAPA and SP) the different screening serological tests target a different cluster of antibodies, some of which are not recognised by the OIE. For instance, the SP test lacks OIE recognition and detects immunoglobulin $M$, immunoglobulin $G_{2}$, and immunoglobulin A (unpublished data, NVSL protocols, Ames Iowa, United States). While the BAPA test has OIE recognition, it detects mainly immunoglobulin $G_{1}$ and immunoglobulin $G_{2}$ and has a sensitivity ranging from $70 \%-99 \%{ }^{44}$ Therefore, the use of any one of the screening tests in isolation could infer non-detection of some of the antibody types. In this study, the screening tests were in total agreement, meaning that at least one of the antibody types they target was present, pointing to possible prior exposure or other cross-reactions, but not infection (as this would be confirmed using the RPPA).

The RPPA test showed that all the test samples from humans and dogs were negative for brucellosis caused by the smooth Brucella strains. Specifically, the canine blood samples were negative for the highly pathogenic canine B. suis. The findings of this study contrasted with those of a similar study that showed a seroprevalence of $7.5 \%$ for human brucellosis in humans using the Card test alone. ${ }^{3}$ The difference could be attributable to the fact that they did not use a quantitative confirmatory serological test (RPPA) alongside the Card test.
Since a positive brucellosis test would necessitate long-term use of antibiotics, ${ }^{45}$ an incorrect brucellosis diagnosis (falsepositive serological reaction) means that patients are placed on unnecessary antibiotics, this in turn aids the development of antibiotic resistance. ${ }^{41}$ Misdiagnosis of the causes of febrile illness in Uganda is a common cause of the misuse of antibiotics. ${ }^{46}$

\section{Limitations}

The serological tests that were used in this study deployed antigens that could not aid the detection of rough colony Brucella spp. such as B. canis and B. ovis. Brucella canis is a species specific to dogs. Therefore, even though the residual samples were negative for B. abortus and B. suis they may not be negative for B. canis.

The Flourescent Polarisation assay is the recommended serological confirmatory test for brucellosis, but it was not used in this study; instead we used a supplemental confirmatory serological test. In this study we did not confirm infection by carrying out a culture for brucellosis.

To the best of our knowledge, the findings of this study can be used as per the scope of the study and in light of its limitations as clearly pointed out.

\section{Conclusion}

This study used the traditional brucellosis screening tests (BAPA, Card and SP) in addition to a quantitative serological confirmatory test (RPPA). The additional use of RPPA confirmed as negative the positive results obtained using the BAPA, SP and Card tests. This implies that the improved specificity on using RPPA might help improve diagnostic accuracy by ruling out false-positive serological reactions. Since this study did not set out to detect B. canis in dogs, but rather canine B. suis, other serological tests specific to $B$. canis would help to complete the brucellosis picture in dogs.

\section{Acknowledgements}

We acknowledge the Norman. E. Borlaug International Fellowship and the National Veterinary Services Laboratory for providing the serological reagents.

\section{Competing interests}

The authors declare that there are no financial or personal relationships that may have had an influence in the writing of this article.

\section{Authors' contributions}

J.B.K. participated in the conception of the idea, data analysis, and writing of the manuscript. T.N. and J.T. participated in the processing of the samples, and writing of the manuscript. 


\section{Sources of support}

Funds for this study were provided by the Norman. E. Borlaug International Fellowship in the East African region.

\section{Data availability statement}

Data is available from the corresponding author upon reasonable request.

\section{Disclaimer}

The views and opinions expressed in this article are those of the authors and do not necessarily reflect the official policy or position of any affiliated agency of the authors.

\section{References}

1. Godfroid J. Brucellosis in livestock and wildlife: Zoonotic diseases without pandemic potential in need of innovative one health approaches. Arch Public Health. 2017;75:34. https://doi.org/10.1186/s13690-017-0207-7

2. Nguna J, Dione $M$, Apamaku $M$, et al. Seroprevalence of brucellosis and risk factors associated with its seropositivity in cattle, goats and humans in Iganga District, Uganda. Pan Afr Med J. 2019;33:99. https://doi.org/10.11604/pamj. 2019.33.99.16960

3. Majalija S, Luyombo P, Tumwine G. Sero-prevalence and associated risk factors of Brucellosis among Malaria negative febrile out-patients in Wakiso district, Central Uganda. BMC Res Notes. 2018;11(1):803. https://doi.org/10.1186/s13104-0183907-3

4. Dutta D, Sen A, Gupta D, et al. Childhood Brucellosis in Eastern India. Indian J Pediatr. 2018; 85, 266-271. https://doi.org/10.1007/s12098-017-2513-z

5. Roushan MR, Amiri MJ. Update on childhood brucellosis. Recent Pat Antiinfect Drug Discov. 2013;8(1):42-46. https://doi.org/10.2174/1574891X11308010008

6. OIE. Handistatus II: Zoonoses (human cases): Global cases of brucellosis in 2004. Paris, France: OIE; 2004.

7. Pappas $G$, Papadimitriou $P$, Akritidis $N$, et al. The new global map of human brucellosis. Lancet Infect Dis. 2006;6(2):91-99. https://doi.org/10.1016/S14733099(06)70382-6

8. Pacheco WA, Genovez ME, Pozzi CR, et al. Excretion of Brucella abortus vaccine B19 strain during a reproductive cycle in dairy cows. Braz J Microbiol. 2012;43(2):594-601. https://doi.org/10.1590/S1517-83822012000200022

9. Sabrina R, Mossadak HT, Bakir M, et al. Detection of Brucella spp. in milk from seronegative cows by real-time polymerase chain reaction in the region of Batna, Algeria. Vet World. 2018;11(3):363-367. https://doi.org/10.14202/vetworld. Algeria. Vet
2018.363-367

10. Islam MS, Islam MA, Khatun MM, et al. Molecular detection of Brucella spp. from milk of seronegative cows from some selected area in Bangladesh. J Pathog. 2018;2018:9378976. https://doi.org/10.1155/2018/9378976

11. Thorsdottir I, Thorisdottir AV. Whole cow's milk in early life. Nestle Nutr Workshop Ser Paediatr Program. 2011;67:29-40. https://doi.org/10.1159/000325573

12. Huber JD, Nicoletti P. Comparison of the results of card, rivanol, complementfixation, and milk ring tests with the isolation rate of Brucella abortus from cattle. Am J Vet Res. 1986;47(7):1529-1531.

13. Alirezalu K, Munekata PES, Parniakov O, et al. Pulsed electric field and mild heating for milk processing: A review on recent advances. J Sci Food Agric 2019;100(1):16-24. https://doi.org/10.1002/jsfa.9942

14. Sfeir MM. Raw milk intake: Beware of emerging brucellosis. J Med Microbiol. 2018;67(5):681-682. https://doi.org/10.1002/jsfa.9942

15. Cossaboom CM, Kharod GA, Salzer JS, et al. Notes from the Field: Brucella abortus vaccine strain RB51 infection and exposures associated with raw milk
consumption - Wise County, Texas, 2017. MMWR Morb Mortal Wkly Rep. 2018;67(9):286. https://doi.org/10.15585/mmwr.mm6709a4

16. Tumwine G, Matovu E, Kabasa JD, et al. Human brucellosis: Sero-prevalence and associated risk factors in agro-pastoral communities of Kiboga District, Central Uganda. BMC Public Health. 2015;15:900. https://doi.org/10.1186/s12889-0152242-z

17. Abd El-Wahab EW, Hegazy YM, El-Tras WF, et al. A multifaceted risk model of brucellosis at the human-animal interface in Egypt. Transboundary and Emerging Diseases. 2019;66(6):2383-2401. https://doi.org/10.1111/tbed.13295

18. Ekpanyaskul C, Santiwattanakul S, Tantisiriwat W, et al. Factors associated with seropositive antibodies to Brucella melitensis in the Nakhon Nayok, Thailand. J Med Assoc Thai. 2012;95 Suppl 12:S40-S46.

19. Mor SM, Wiethoelter AK, Lee A, et al. Emergence of Brucella suis in dogs in New South Wales, Australia: Clinical findings and implications for zoonotic transmission. BMC Vet Res. 2016;12(1):199. https://doi.org/10.1186/s12917-016-0835-0
20. Wareth G, Melzer F, El-Diasty M, et al. Isolation of Brucella abortus from a dog and a cat confirms their biological role in re-emergence and dissemination of Bovine Brucellosis on dairy farms. Transbound Emerg Dis. 2017;64(5):e27-e30. https:// Brucellosis on dairy farms. Tr
doi.org/10.1111/tbed.12535

21. Trampuz A, Jereb M, Muzlovic I, et al. Clinical review: Severe malaria. Crit Care. 2003;7(4):315-323. https://doi.org/10.1186/cc2183

22. Alemnji GA, Zeh C, Yao K, et al. Strengthening national health laboratories in subSaharan Africa: A decade of remarkable progress. Trop Med Int Health. 2014;19(4):450-458. https://doi.org/10.1111/tmi.12269

23. Migisha R, Dan N, Boum Y, et al. Prevalence and risk factors of brucellosis among febrile patients attending a community hospital in south western Uganda. Sci Rep. 2018;8(1):15465. https://doi.org/10.1038/s41598-018-33915-9

24. Bozdemir SE, Altintop YA, Uytun S, et al. Diagnostic role of mean platelet volume and neutrophil to lymphocyte ratio in childhood brucellosis. Korean J Intern Med. 2017;32(6):1075-1081. https://doi.org/10.3904/kjim.2016.092

25. Ducrotoy MJ, Ammary K, Ait Lbacha $\mathrm{H}$, et al. Narrative overview of animal and human brucellosis in Morocco: Intensification of livestock production as a driver for emergence? Infect Dis Poverty. 2015;4:57. https://doi.org/10.1186/s40249015-0086-5

26. Brennan A, Cross PC, Portacci K, et al. Shifting brucellosis risk in livestock coincides with spreading seroprevalence in elk. PLoS One. 2017;12(6):e0178780. https:// doi.org/10.1371/journal.pone.0178780

27. Hubbard K, Wang M, Smith DR. Seroprevalence of brucellosis in Mississippi shelter dogs. Prev Vet Med. 2018;159:82-86. https://doi.org/10.1016/j. prevetmed.2018.09.002

28. Ducrotoy MJ, Bardosh KL. How do you get the Rose Bengal Test at the point-ofcare to diagnose brucellosis in Africa? The importance of a systems approach. Acta Trop. 2017;165:33-39. https://doi.org/10.1016/j.actatropica.2016.10.004

29. Orduña A, Almaraz A, Prado A, et al. Evaluation of an immunocaptureagglutination test (Brucellacapt) for serodiagnosis of human brucellosis. J Clin Microbiol. 2000;38(11):4000-4005. https://doi.org/10.1128/JCM.38.11.40004005.2000

30. Diaz R, Casanova A, Ariza J, Moriyon I. The Rose Bengal test in human brucellosis: A neglected test for the diagnosis of a neglected disease. PLoS Negl Trop Dis. 2011;5(4):e950. https://doi.org/10.1371/journal.pntd.0000950

31. Muloki HN, Erume J, Owiny DO, et al. Prevalence and risk factors for brucellosis in prolonged fever patients in post-conflict Northern Uganda. Afr Health Sci. 2018;18(1):22-28. https://doi.org/10.4314/ahs.v18i1.4

32. Tumwine G, Matovu E, Kabasa JD, et al. Human brucellosis: Sero-prevalence and associated risk factors in agro-pastoral communities of Kiboga District, Central Uganda. BMC Publ Health. 2015;15:900.

33. Erume J, Roesel K, Dione MM, et al. Serological and molecular investigation for brucellosis in swine in selected districts of Uganda. Trop Anim Health Prod. 2016;48(6):1147-1155

34. Hall SM, Confer AW, Tabatabai LB, et al. Detection of serum antibody to Brucella abortus in cattle by use of a quantitative fluorometric immunoassay. J Clin Microbiol. 1984;20(6):1023-1027.

35. Bejon P, Andrews L, Hunt-Cooke A, et al. Thick blood film examination for Plasmodium falciparum malaria has reduced sensitivity and underestimates parasite density. Malar J. 2006;5:104

36. Dean AG SK, Soe MM. OpenEpi: Open source epidemiologic statistics for public health, Version 3 [homepage on the Internet]. n.d. [updated 2013 June 04; cited 2019 Aug 15]. Available from: www.OpenEpi.com

37. Assenga JA, Matemba LE, Muller SK, et al. Epidemiology of Brucella infection in the human, livestock and wildlife interface in the Katavi-Rukwa ecosystem, Tanzania. BMC Vet Res. 2015;11:189.

38. Acosta-González RI, González-Reyes I, Flores-Gutiérrez GH. Prevalence of Brucella abortus antibodies in equines of a tropical region of Mexico. Can J Vet Res. 2006;70(4):302-304.

39. Ducrotoy MJ, Bertu WJ, Ocholi RA, et al. Brucellosis as an emerging threat in developing economies: Lessons from Nigeria. PLoS NegI Trop Dis. 2014;8(7):e3008.

40. Njeru J, Wareth G, Melzer F, et al. Systematic review of brucellosis in Kenya: Disease frequency in humans and animals and risk factors for human infection. BMC Publ Health. 2016;16(1):853.

41. De Glanville WA, Conde-Álvarez R, Moriyón I, et al. Poor performance of the rapid test for human brucellosis in health facilities in Kenya. PLoS Negl Trop Dis. 2017;11(4):e0005508.

42. Ebani VV, Cerri D, Fratini F, Bey RF, Andreani E. Serological diagnosis of brucellosis caused by Brucella canis. New Microbiol. 2003;26(1):65-73.

43. Bonfini B, Chiarenza G, Paci V, et al. Cross-reactivity in serological tests for brucellosis: A comparison of immune response of Escherichia coli 0157:H7 and Yersinia enterocolitica 0:9 vs Brucella spp. Vet Ital. 2018;54(2):107-114.

44. OIE. Manual of diagnostic tests and vaccines for terrestrial animals [homepage on the Internet]. 2018 [cited 2019 Oct 15]. Available from: https://www.oie.int/ international-standard-setting/terrestrial-manual

45. Yuan MJ, Li SH, Huang $Y$, et al. Diagnosis and treatment of seven patients with brucellosis in non-pastoral areas. Zhonghua Nei Ke Za Zhi. 2019;58(8): 596-598.

46. Batwala V, Magnussen $P$, Nuwaha F. Antibiotic use among patients with febrile illness in a low malaria endemicity setting in Uganda. Malar J. 2011;10:377. 